\title{
Manejo da irrigação, inoculação e nitrogênio no feijoeiro de inverno
}

\author{
Irrigation management, inoculation and nitrogen in dry bean crop \\ Manejo de riego, inoculación y nitrógeno en frijol de invierno
}

Recebido: 21/06/2021 | Revisado: 28/06/2021 | Aceito: 08/07/2021 | Publicado: 18/07/2021

Marcos Jefferson Kraeski

ORCID: https://orcid.org/0000-0002-1026-8057

Universidade Estadual de Mato Grosso do Sul, Brasil

E-mail: marcoskraeski@gmail.com

Adriano da Silva Lopes

ORCID: https://orcid.org/0000-0003-2211-6576

Universidade Estadual de Mato Grosso do Sul, Brasil

E-mail: lopes@uems.br

Eder Duarte Fanaya Júnior

ORCID: https://orcid.org/0000-0002-2292-6504

Universidade Estadual de Mato Grosso do Sul, Brasil

E-mail: eder.fanayajr@alumni.usp.br

Arthur Pacheco

ORCID: https://orcid.org/0000-0001-8387-3461 Universidade Estadual de Mato Grosso do Sul, Brasil

E-mail: arthur_ap@hotmail.com

Michell Arce Centurião

ORCID: https://orcid.org/0000-0003-2745-3504 Universidade Estadual de Mato Grosso do Sul, Brasil

E-mail: michellac999@gmail.com

Ana Caroline Mello Arevalo

ORCID: https://orcid.org/0000-0002-1277-1553 Universidade Estadual de Mato Grosso do Sul, Brasil

E-mail: amelloarevalo@gmail.com

Adriano de França

ORCID: https://orcid.org/0000-0001-6710-0282 Universidade Estadual de Mato Grosso do Sul, Brasil

E-mail: adrianogll@hotmail.com

Rosevaldo Domingos Medeiros

ORCID: https://orcid.org/0000-0003-1540-0931

Universidade Estadual de Mato Grosso do Sul, Brasil

E-mail: rosevaldomdrrdm@gmail.com

\section{Resumo}

Entre os fatores que influenciam na produtividade do feijoeiro, a precipitação e a nutrição são consideradas limitantes. O objetivo deste trabalho foi avaliar o manejo da irrigação, adubação nitrogenada e a inoculação de sementes, no feijoeiro de inverno, em Aquidauana - MS. O trabalho foi realizado na área experimental da Universidade Estadual de Mato Grosso do Sul, utilizando a cultivar TAA Dama com semeadura em 24 de maio de 2018. O delineamento experimental foi em blocos casualizados, em esquema de parcelas subsubdivididas, com quatro repetições. As parcelas foram os manejos de irrigação Penman-Monteith e tensiometria. As subparcelas foram com e sem inoculação de Rhizobium tropici semia 4077 e, nas subsubparcelas, foram empregadas as doses de nitrogênio em cobertura (50, 100, 150 e $200 \mathrm{~kg} \mathrm{ha}^{-1}$ e um com ausência de nitrogênio). Foram avaliados o número e massa seca de nódulos, número de vagens por planta, massa média de 100 grãos, número de grãos por vagens, produtividade de grãos, eficiência no uso da água. Maiores doses de adubação nitrogenada em cobertura proporcionam maiores produtividades de grãos e eficiência no uso da água pelo feijoeiro. A presença ou ausência de inoculação não influencia na produtividade de grãos do feijoeiro irrigado.

Palavras-chave: Eficiência do uso da água, Penman-Monteith, Phaseolus vulgaris L., Rhizobium tropici, Tensiômetros.

\section{Abstract}

Among the factors that influence bean productivity, precipitation and nutrition are considered limiting factors. The study aim was to evaluate the irrigation management, Nitrogen fertilization and seed inoculation, in dry bean, in Aquidauana-MS. The work was carried out in the experimental area of the State University of Mato Grosso do Sul, Aquidauana campus, using the cultivar TAA Dama sowed on May 24 $4^{\text {th }}, 2018$. The experimental design was in randomized blocks, in a split-plot scheme, with four replications. The plots were the Penman-Monteith model 
irrigation and tensiometry managements. The subplots were with and without Rhizobium tropici semia 4077 inoculation and, in the sub-subplots, topdressing Nitrogen doses $\left(50,100,150\right.$ and $200 \mathrm{~kg} \mathrm{ha}^{-1}$ and one without Nitrogen) were used. The number and dry matter of nodules were evaluated, pods number per plant, average mass of 100 grains, grains number per pod, grain yield, water use efficiency. Higher Nitrogen doses topdressing provide higher grain yields and water use efficiency by the dry bean. The inoculation presence or absence doesn't influence the grain irrigated bean yield.

Keywords: Penman-Monteith, Phaseolus vulgaris L., Rhizobium tropici, Tensiometry, Water use efficiency.

\section{Resumen}

Entre los factores que influyen en la productividad del frijol, la precipitación y la nutrición se consideran factores limitantes. El objetivo de este trabajo fue evaluar el manejo del riego, fertilización nitrogenada e inoculación de semillas, en frijol de invierno, en Aquidauana - MS. El trabajo se realizó en el área experimental de la Universidad Estatal de Mato Grosso do Sul, utilizando el cultivar TAA Dama con siembra el 24 de mayo de 2018. El diseño experimental fue en bloques al azar, en un esquema de parcelas divididas, con cuatro réplicas. Las parcelas fueron las gestiones de regadío y tensiometría de Penman-Monteith. Las subparcelas fueron con y sin inoculación de Rhizobium tropici semia 4077 y, en las subparcelas, se utilizaron dosis de cobertura de nitrógeno (50,100, 150 y $200 \mathrm{~kg} \mathrm{ha}^{-1}$ y una sin nitrógeno). Se evaluó el número y masa seca de nódulos, número de vainas por planta, masa promedio de 100 granos, número de granos por vaina, rendimiento de grano, eficiencia en el uso del agua. Las dosis más altas de cobertura de nitrógeno proporcionan mayores rendimientos de grano y eficiencia en el uso del agua por parte del frijol común. La presencia o ausencia de inoculación no influye en el rendimiento de grano del frijol común de regadío.

Palabras clave: Eficiencia en el uso del agua, Penman-Monteith, Phaseolus vulgaris L., Rhizobium tropici, Tensiómetros.

\section{Introdução}

O feijão (Phaseolus vulgaris L.) é um alimento de grande importância na dieta da população brasileira, constituindo uma das principais fontes de proteína, sendo uma das mais representativas explorações agrícolas, a nível nacional (CONAB, 2019). Entretanto, a variabilidade climática é um dos fatores determinantes para a produtividade, mantendo-se assim abaixo dos patamares desejados.

Períodos de temperaturas superiores a $35^{\circ} \mathrm{C}$ afetam a cultura em diferentes estádios fenológicos, principalmente no florescimento e na frutificação, causando abscisão de flores e redução do número de grãos por vagem, e na massa desses grãos (Pereira et al., 2014). Já, períodos de baixa precipitação ou de déficit hídrico, influenciam diretamente no número de vagens por planta, número de grãos por vagens e na massa dos grãos, comprometendo a produtividade (Conceição et al., 2018).

Entre as culturas cultivadas em maior quantidade, o feijoeiro é o mais sensível ao déficit hídrico. Isso pode estar relacionado ao seu ciclo menor e ao seu metabolismo (Viçosi et al., 2017). O cultivo no período de inverno, em razão do índice pluviométrico ser menor em relação ao verão, faz da irrigação uma ferramenta fundamental para a produção. Fernandes (2012) ressalta que a resposta financeira atrativa neste período, devido a menor oferta no mercado, desperta o interesse de agricultores que adotam tecnologias avançadas na produção.

Para Drugowich e D'Auria (2017), o manejo da água na irrigação de cultivos é o principal mecanismo de otimização do seu uso na agricultura. Assim sendo, o manejo inadequado da irrigação pode proporcionar prejuízos referentes ao desperdício de água e energia, além da lixiviação de nutrientes do solo, que pode reduzir a produtividade de grãos do feijoeiro.

Em sistemas de cultivo irrigado, a cultura tem condições de expressar melhor seu potencial produtivo. Aliado a isso, a nutrição delas também deve ser considerada. Ramires et al. (2018) destacam que a produtividade de grãos de feijoeiro foi dependente da interação da cultivar e de doses de nitrogênio (N), enquanto Sabundjian et al. (2013) ressaltam que o N é um dos nutrientes absorvidos em maior quantidade, em todas as fases de desenvolvimento.

Uma alternativa para a redução dos custos com a aquisição de adubos nitrogenados é por meio da fixação biológica de $\mathrm{N}$ (FBN) que, em condições favoráveis, pode fornecer até $80 \mathrm{~kg} \mathrm{ha}^{-1} \mathrm{de} \mathrm{N}$ para a cultura (Rebeschini et al., 2014).

Embora a eficiência da FBN no feijoeiro seja considerada variável, de acordo com Santana et al. (2018) a inoculação de sementes pode reduzir ou até mesmo substituir o $\mathrm{N}$ em cobertura, mantendo a produtividade de grãos, evidenciando sua 
importância para obtenção de maior rentabilidade do feijoeiro. A falta de respostas à inoculação com Rhizobium tropici foi relacionada à incapacidade dessas estirpes inoculadas competirem com espécies nativas, limitando assim a responsividade da inoculação (BRITO et al., 2015).

Com base na importância de estudos capazes de gerar informações sobre as necessidades hídricas e nutricionais do feijoeiro cultivado em época de inverno, espera-se que a inoculação de sementes com Rhizobium tropici possa reduzir ou até mesmo substituir a necessidade de adubação nitrogenada, que proporcione maior produtividade para a cultura, especialmente sob condição de irrigação.

Diante disso, o objetivo deste trabalho foi avaliar os efeitos dos manejos de irrigação, inoculação de sementes e nitrogênio em cobertura no desenvolvimento do feijoeiro de inverno, em Aquidauana-MS.

\section{Metodologia}

O trabalho foi conduzido na área experimental da Universidade Estadual de Mato Grosso do Sul (UEMS), em

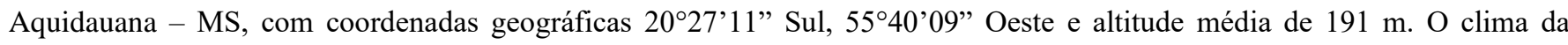
região, segundo a classificação de Köppen, é descrita como Aw, definido como clima tropical quente subúmido, com estação chuvosa no verão e seca no inverno (Alvares et al., 2014) e precipitação média anual ${ }^{1}$ de $1.282,7 \mathrm{~mm}$. O solo foi identificado como Argissolo Vermelho distrófico (Schiavo et al., 2010).

Os dados climáticos monitorados diariamente por meio da estação meteorológica da UEMS, foram: temperaturas, precipitação, radiação solar, velocidade do vento e umidade relativa do ar, bem como foi calculado o somatório de graus-dia (GD), em que se tomou como temperatura basal inferior $10{ }^{\circ} \mathrm{C}$ e temperatura basal superior $35^{\circ} \mathrm{C}$ (Miranda, Campelo Junior, 2010).

A temperatura máxima registrada foi de $34,5{ }^{\circ} \mathrm{C}$ aos $78 \mathrm{DAE}$ e a mínima foi de $7,0{ }^{\circ} \mathrm{C}$ aos $70 \mathrm{DAE}$, enquanto a temperatura média no período foi de $21,6^{\circ} \mathrm{C}$ (Figura 1). O florescimento ocorreu com 443,8 GD e a maturação fisiológica com 927,9 GD, corroborando os resultados de Teixeira, Stone e Heinemann (2015) que encontraram valores semelhantes para cultivares de crescimento indeterminado.

Figura 1. Temperaturas média, mínima e máxima $\left({ }^{\circ} \mathrm{C}\right)$ do ar, graus-dia acumulados ao longo do ciclo da cultura (dias após a emergência - DAE), para as fases fenológicas de desenvolvimento vegetativo (DV), florescimento e enchimento de grãos (FEG) e maturação (M) - feijão de inverno (cultivar TAA Dama).

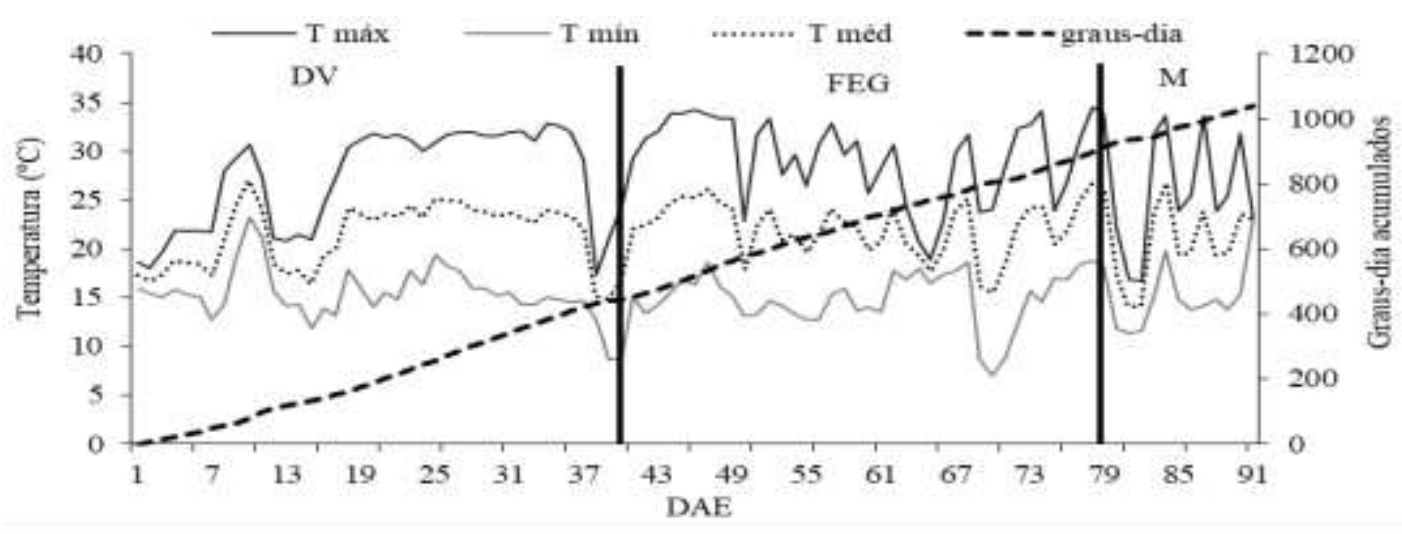

Fonte: Autores.

O experimento foi realizado sob o sistema de irrigação por pivô central, o qual foi avaliado quanto a sua uniformidade

${ }^{1}$ Pluviosidade média calculada a partir dos dados do ano de 2007 à 2018, obtida em: http://www.inmet.gov.br/portal/i ndex.php?r=estacoes/estacoesautomaticas 
de aplicação de água (Figura 2), utilizando o Coeficiente de Uniformidade de Christiansen (CUC) e Coeficiente de Uniformidade de Distribuição (CUD) (Bernardo et al., 2006).

Figura 2. Lâmina média e observada durante o teste de uniformidade do pivô central a $100 \%$ e $50 \%$ de sua velocidade máxima.

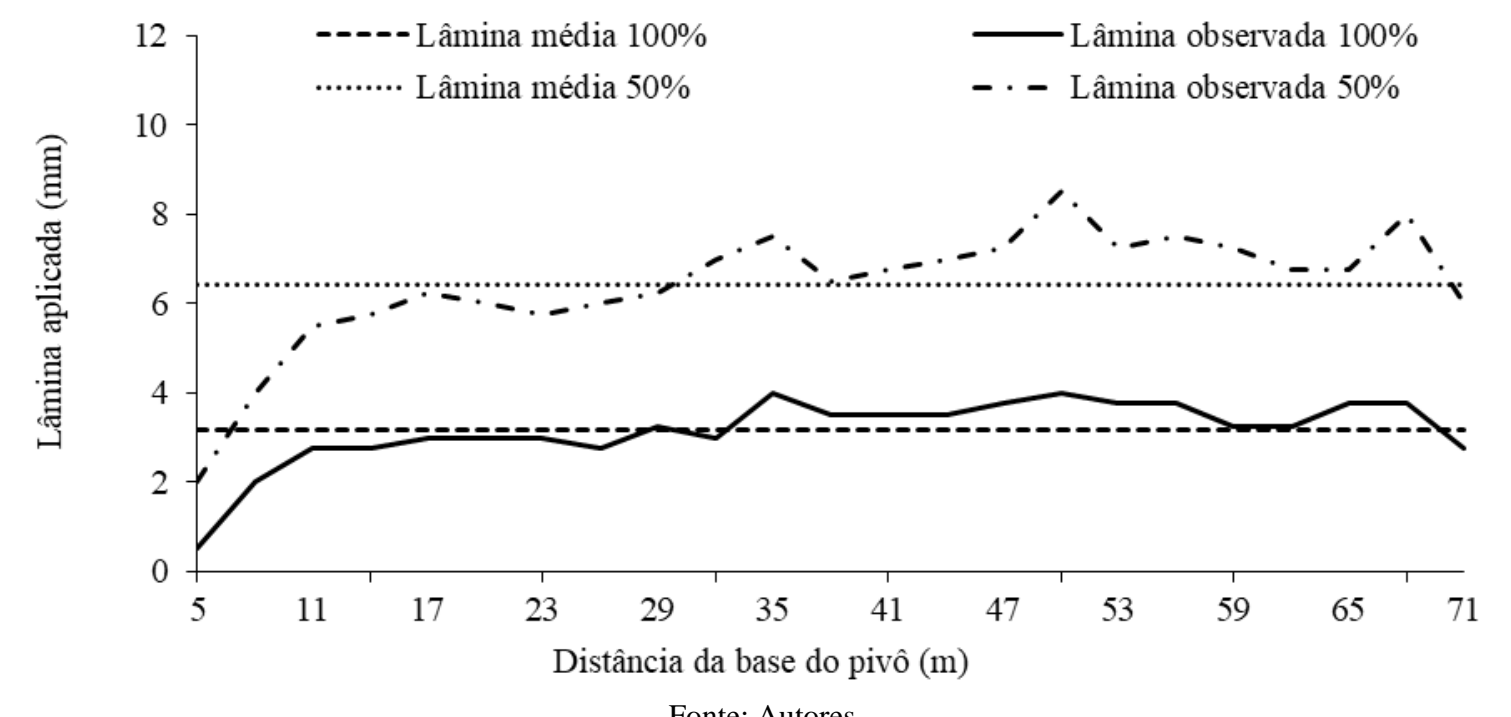

Para a velocidade de $100 \%$, o resultado do CUC foi de $87,1 \%$ e do CUD de $84,3 \%$. Já, para a velocidade de $50 \%$, os resultados obtidos foram de 88,9\% para o CUC e de 90,8\% para o CUD. Esses resultados configuram boa distribuição da lâmina de água aplicada ao longo da área do equipamento utilizado.

O delineamento experimental foi em blocos casualizados, com 20 tratamentos e 04 repetições, totalizando 80 unidades experimentais. Cada parcela experimental consistiu por 04 linhas de plantas com $05 \mathrm{~m}$ de comprimento, sendo que a área útil de cada parcela compreendeu duas linhas centrais por $04 \mathrm{~m}$ de comprimento, totalizando $3,6 \mathrm{~m}^{2}$.

As parcelas foram constituídas pelos manejos de irrigação: Penman-Monteith (PM) e tensiometria (TENS). As subparcelas foram constituídas pelo uso ou não de inoculação na semeadura com Rhizobium tropici semia 4077 e, nas subsubparcelas, foram empregadas as doses de $\mathrm{N}$ em cobertura (ureia -45\% N) no estádio V4: 50, 100, 150 e $200 \mathrm{~kg} \mathrm{ha}^{-1} \mathrm{e}$ um com ausência de N. Após a adubação em cobertura aplicou-se lâmina de água de 10,2 mm.

Para o tratamento de sementes, utilizou-se o fungicida Carboxina + Thiram na dose $18+18 \mathrm{~g}$ i.a. para $30 \mathrm{~kg}$ de sementes, sendo feita um dia antes da semeadura e, a inoculação com Rhizobium tropici na dose de $4 \mathrm{~mL} \mathrm{~kg}^{-1}$ no dia da semeadura.

A semeadura foi realizada em sistema convencional em 24 de maio de 2018, utilizando a cultivar TAA Dama, com espaçamento entrelinhas de $0,45 \mathrm{~m}$ e estande médio de 10 plantas por metro, cuja adubação por ocasião da semeadura foi realizada com o adubo formulado 04-20-20, em todas as parcelas, na dose de $168 \mathrm{~kg} \mathrm{ha}^{-1}$, de acordo com Sousa e Lobato (2004), cuja análise química do solo encontra-se na Tabela 1.

Tabela 1. Análise química do solo na profundidade de 0 a $0,2 \mathrm{~m}$.

\begin{tabular}{|c|c|c|c|c|c|c|c|c|c|c|}
\hline \multirow{2}{*}{$\mathrm{pH}^{*}$} & \multirow{2}{*}{$\begin{array}{c}\mathrm{P} \\
\mathrm{mg} \mathrm{dm}{ }^{-3}\end{array}$} & \multirow{2}{*}{$\begin{array}{c}\text { M.O } \\
\%\end{array}$} & $\mathrm{~K}$ & $\mathrm{Ca}$ & $\mathrm{Mg}$ & $\mathrm{Al}$ & $\mathrm{H}+\mathrm{Al}$ & SB & $\mathrm{T}$ & $\mathrm{V}$ \\
\hline & & & \multicolumn{7}{|c|}{$\mathrm{cmol}_{\mathrm{c}} \mathrm{dm}^{-3}$} & $\%$ \\
\hline 6,1 & 47,7 & 22,3 & 0,39 & 3,8 & 1 & 0 & 3,3 & 5,18 & 8,48 & 61 \\
\hline
\end{tabular}

*pH em água 1:2,5, Matéria Orgânica (M.O), Teor de argila menor que 15\%, Soma de bases (SB), CTC a pH 7,0 (T), Saturação de bases (V), P e K: Extrator Mehlich. Fonte: Autores. 
O manejo da irrigação utilizando o modelo de PM, este recomendado pela Organização das Nações Unidas para a Alimentação e a Agricultura (FAO) como método padrão e foi baseado na estimativa da evapotranspiração de referência (ETo) segundo Allen et al. (1998), com fator "p" de depleção de água no solo de 0,5. Com o valor de ETo, foi estimada a evapotranspiração da cultura (ETc), conforme Equação 1.

"ETc=ETo x Kc"

Em que: ETc $=$ Evapotranspiração da cultura $\left(\mathrm{mm} \mathrm{d}^{-1}\right)$, ETo $=$ Evapotranspiração de referência $\left(\mathrm{mm} \mathrm{d}^{-1}\right), \mathrm{Kc}=$ Coeficiente da cultura (adimensional), o qual utilizou valores de 0,40 para fase inicial e desenvolvimento vegetativo, 1,15 para fase de floração e enchimento de grãos, e 0,35 para a fase de maturação (ALLEN et al., 1998).

No manejo da irrigação baseado na TENS, foram instalados conjuntos de tensiômetros a 0,15 m, 0,30 m e 0,45 m de profundidade, tendo como referência a profundidade de $0,15 \mathrm{~m}$ para a tomada de decisão, enquanto os demais tensiômetros para monitoramento da umidade ao longo do perfil do solo. O momento de se proceder à irrigação foi definido quando a média das leituras apresentavam valores de tensão da água no solo igual ou superior a $40 \mathrm{kPa}$ (SILVEIRA, STONE \& MOREIRA, 2001). A lâmina de irrigação ou água disponível consumida até o dia da irrigação (LLi), em mm, foi calculada conforme a equação 2:

"LLi=" ("Өcc- $\theta \mathrm{a} "$ )"1000 Z"

Em que: " $\theta$ " cc $=$ umidade do solo na capacidade de campo $\left(\mathrm{m}^{3} \mathrm{~m}^{-3}\right), " \theta^{\prime} \mathrm{a}=$ umidade atual do solo $\left(\mathrm{m}^{3} \mathrm{~m}^{-3}\right), \mathrm{Z}=$ profundidade efetiva do sistema radicular (m), o qual utilizou valores de 0,1 m (1 a 9 DAE), 0,2 m (10 a 33 DAE), 0,3 m (34 a 44 DAE) e 0,4 m (44 a 91 DAE).

Foi aplicado o inseticida Tiametoxam + Lambda-cialotrina na dose de 42,3 + 31,8 g i.a. ha ${ }^{-1}$ para controle de mosca branca. E uma aplicação preventiva do fungicida Trifloxistrobina + tebuconazol no estádio V4, na dose de $75+150 \mathrm{~g}$ i.a. ha ${ }^{-1}$. As plantas invasoras foram controladas por capinas manuais aos 14 dias após a emergência (DAE) e aplicação dos herbicidas fluazifope-p-butílico e fomesafem ambos na dose de $250 \mathrm{~g}$ i.a. ha ${ }^{-1}$.

Quando a cultura estava em estádio R5, foram coletadas 05 plantas de cada parcela, realizando as avaliações do número de nódulos por planta (NNP) e a massa seca de nódulos (MSN) (Silva et al., 2009). Ao final do ciclo do feijoeiro todas as plantas da área útil foram contadas, bem como o número total de vagens produtivas, estimando assim o número de vagens por planta (NVP).

Para a massa de 100 grãos (MCG) separou-se, da produção de grãos colhidas na área útil de cada unidade experimental, 3 amostras aleatórias que foram pesadas em balança de precisão de 0,01 g e depois determinadas as suas umidades para correção para $13 \%$ em base úmida. Com os dados de número de vagens e da massa total de grãos, foi estimado o número de grãos por vagem (NGV) e a produtividade de grãos (PROD) em $\mathrm{kg} \mathrm{ha}^{-1}$ a partir da pesagem da massa total de cada unidade experimental.

A eficiência no uso da água (EUA) $\left(\mathrm{kg} \mathrm{m}^{-3}\right)$ foi calculada por meio da razão entre a PROD $\left(\mathrm{kg} \mathrm{ha}^{-1}\right)$ e a lâmina total aplicada (mm) convertida em volume de água para todo o ciclo da cultura $\left(\mathrm{m}^{3} \mathrm{ha}^{-1}\right)$.

A natureza da pesquisa foi quantitativa e seguiu a metodologia estabelecida por Allen et al. (1998) e Silveira, Stone \& Moreira (2001). Para a análise dos dados, foi realizada uma análise de variância para todas as características observadas. Logo 
após, procedeu-se o teste de médias para os manejos de irrigação e inoculação de sementes, utilizando o teste Tukey a 5\% de probabilidade. Já para as doses de N, foi realizada análise de regressão.

\section{Resultados e Discussão}

Foram registrados $87,2 \mathrm{~mm}$ de precipitação pluviométrica durante o ciclo do feijoeiro, conforme pode ser observado mal distribuídos ao longo de seu crescimento e desenvolvimento, sendo que até a metade da fase de enchimento de grãos ocorreu uma chuva, com volume de $13,3 \mathrm{~mm}$. A evapotranspiração média do período foi de $2,31 \mathrm{~mm} \mathrm{~d}^{-1}$ evidenciando a necessidade de irrigação para complementar a demanda hídrica da cultura e disponibilizar água com maior uniformidade ao longo do ciclo (Figura 3).

Figura 3. Variação do armazenamento de água no solo para o manejo por tensiômetros até $0,40 \mathrm{~m}$ de profundidade, assim como as lâminas de irrigação aplicadas e de chuva recebida pelas plantas de feijoeiro. Em que: água facilmente disponível (AFD), água disponível (AD), para as fases fenológicas de desenvolvimento vegetativo (DV), florescimento e enchimento de grãos (FEG) e maturação (M).

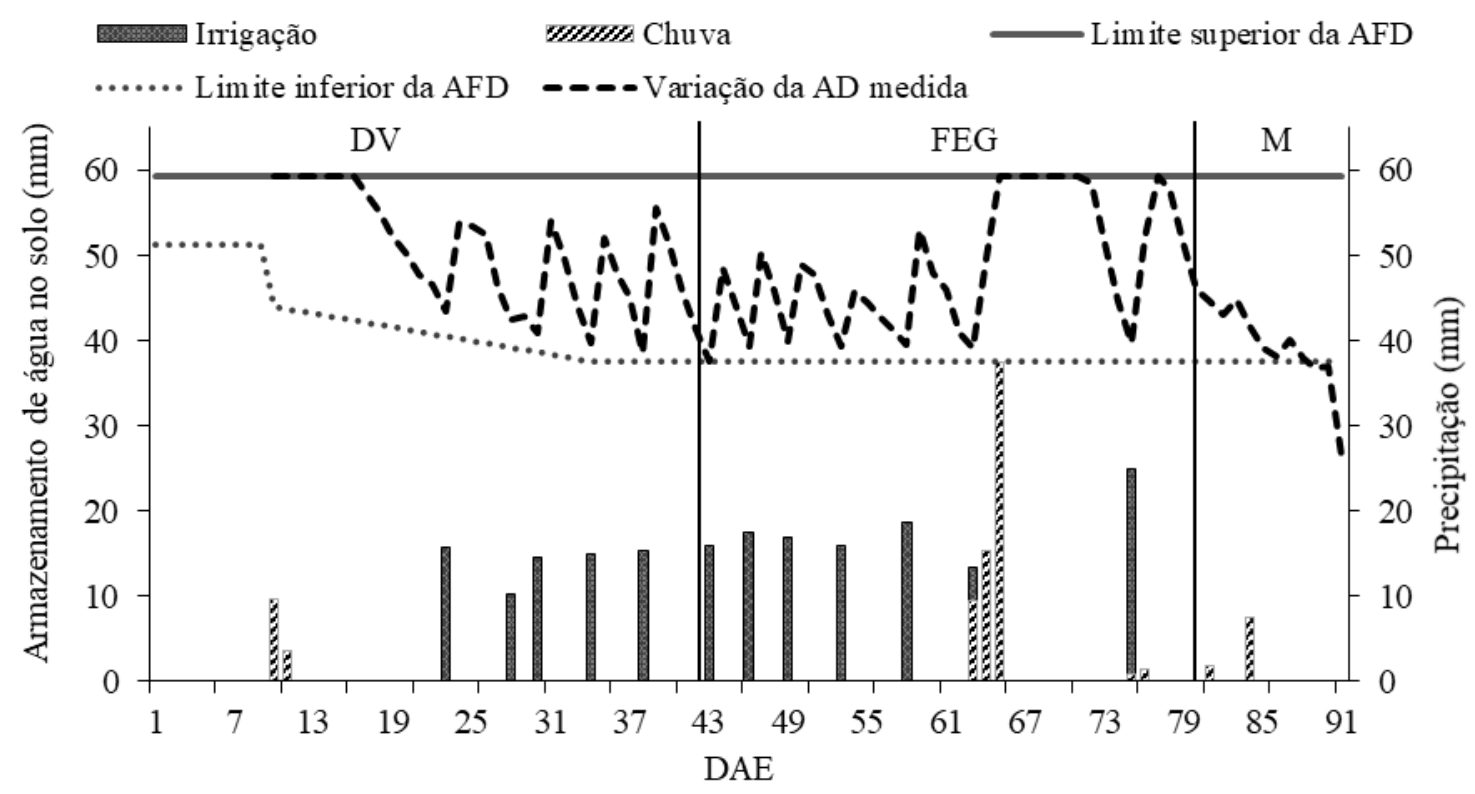

Fonte: Autores.

Bastos et al. (2016) ressaltam que restrições hídricas durante o desenvolvimento vegetativo e enchimento de grãos no feijoeiro proporcionam redução na produtividade da cultura.

As lâminas totais (precipitação + irrigação) foram de 281,5 mm para TENS e de 237,1 mm para PM. Fietz et al. (2018) utilizando manejo de irrigação por PM obtiveram resultados semelhantes com lâmina total de 243,8 mm. O manejo de irrigação por TENS estimou, em média, quantidade total de água 18,7\% maior do que o PM, ficando na faixa ideal de acordo com Oliveira et al. (2018), que é de 250 a 350 mm distribuídos durante seu ciclo. Os manejos de irrigação por PM e TENS proporcionaram água disponível dentro do limite da água facilmente disponível.

Testezlaf (2017) relatou que além de atender a demanda hídrica do ciclo da cultura, a disposição temporal é outro fator importante e, nesse sentido, no manejo da irrigação realizado por TENS, a variação de água disponível no solo ficou dentro dos limites da água facilmente disponível (AFD), abaixo apenas quando a cultura já havia atingido a maturação fisiológica e a irrigação suspensa, evidenciando que a demanda hídrica da cultura foi atendida, tanto em quantidade, quanto em distribuição ao longo de seu ciclo. 
No manejo da irrigação estimado por PM, o volume de água fornecido para a cultura ficou próximo a demanda da cultura e, também, acima do limite da AFD. Embora quando confrontado com os dados oriundos do TENS (Figura 4), ficou abaixo do limite inferior da AFD apenas em alguns momentos, o que indica que o modelo de PM também é eficiente para estimativa de dotação hídrica para o feijoeiro.

Figura 4. Variação do armazenamento de água no solo para o manejo por Penman-Monteith até $0,40 \mathrm{~m}$ de profundidade, e a variação de água disponível medida por tensiômetros, assim como as lâminas de irrigação aplicadas e de chuva recebida pelas plantas de feijoeiro. Em que: água facilmente disponível (AFD), água disponível (AD), para as fases fenológicas de desenvolvimento vegetativo (DV), florescimento e enchimento de grãos (FEG) e maturação (M).

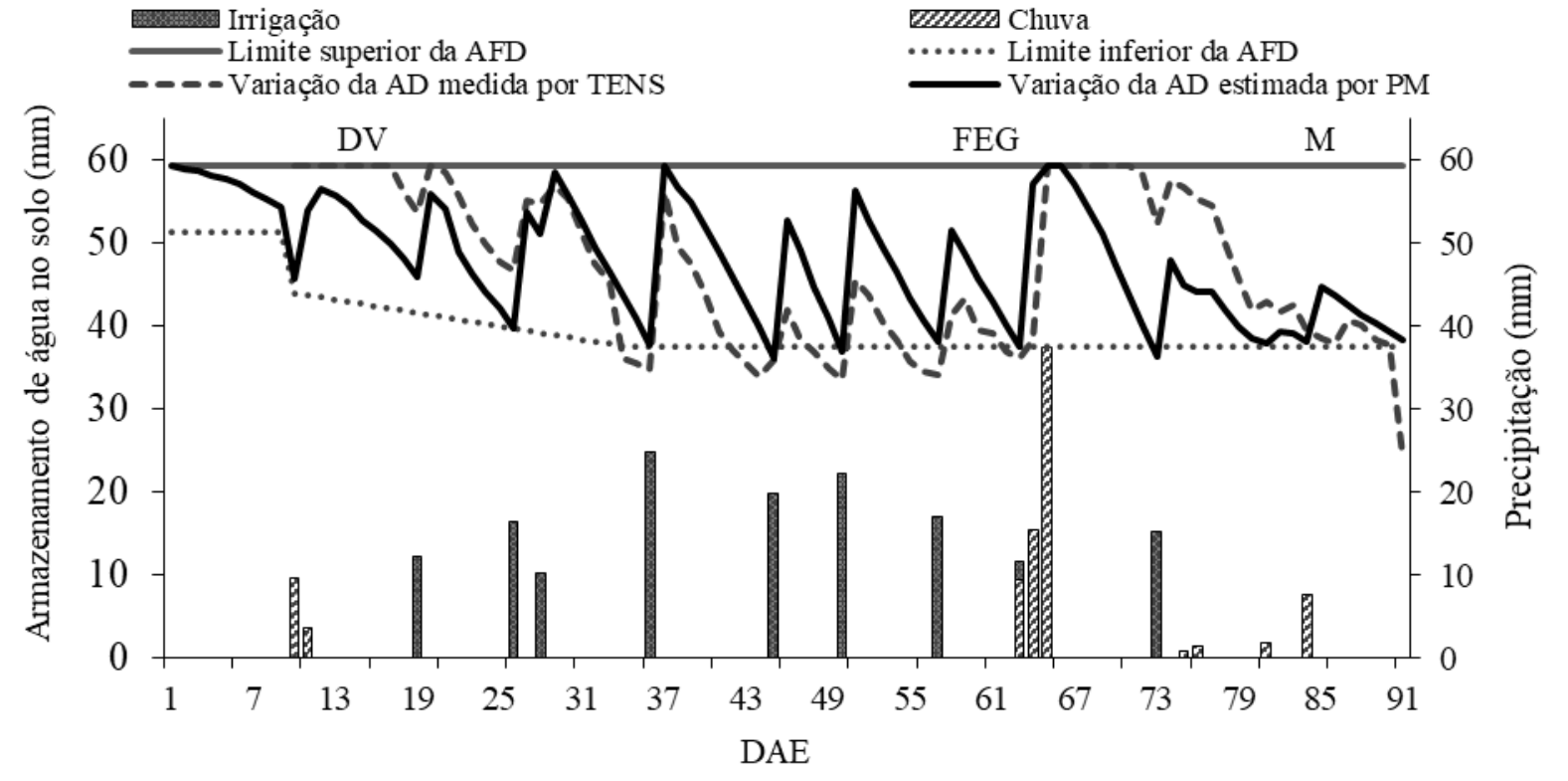

Fonte: Autores.

O manejo da irrigação por TENS proporcionou melhores resultados que por PM, para as variáveis NVP e NGV (Tabela 2), isso pode estar relacionado a maior disponibilidade de água deste manejo, principalmente entre os estádios fenológicos R7 e R8, onde o PM na comparação com TENS (Figura 4), proporcionou momentos em que a AD ficou abaixo da AFD, podendo ter influenciado diretamente nessas variáveis, já que restrições hídricas podem reduzir parâmetros relacionados a produtividade (Bastos et al., 2016). 
Tabela 2. Número de nódulos por planta (NNP), massa seca de nódulos (MSN), massa de 100 grãos (MCG), número de vagens por planta (NVP), número de grãos por vagem (NGV), produtividade de grãos (PROD) e eficiência do uso da água (EUA), em função dos tratamentos e análise de variância.

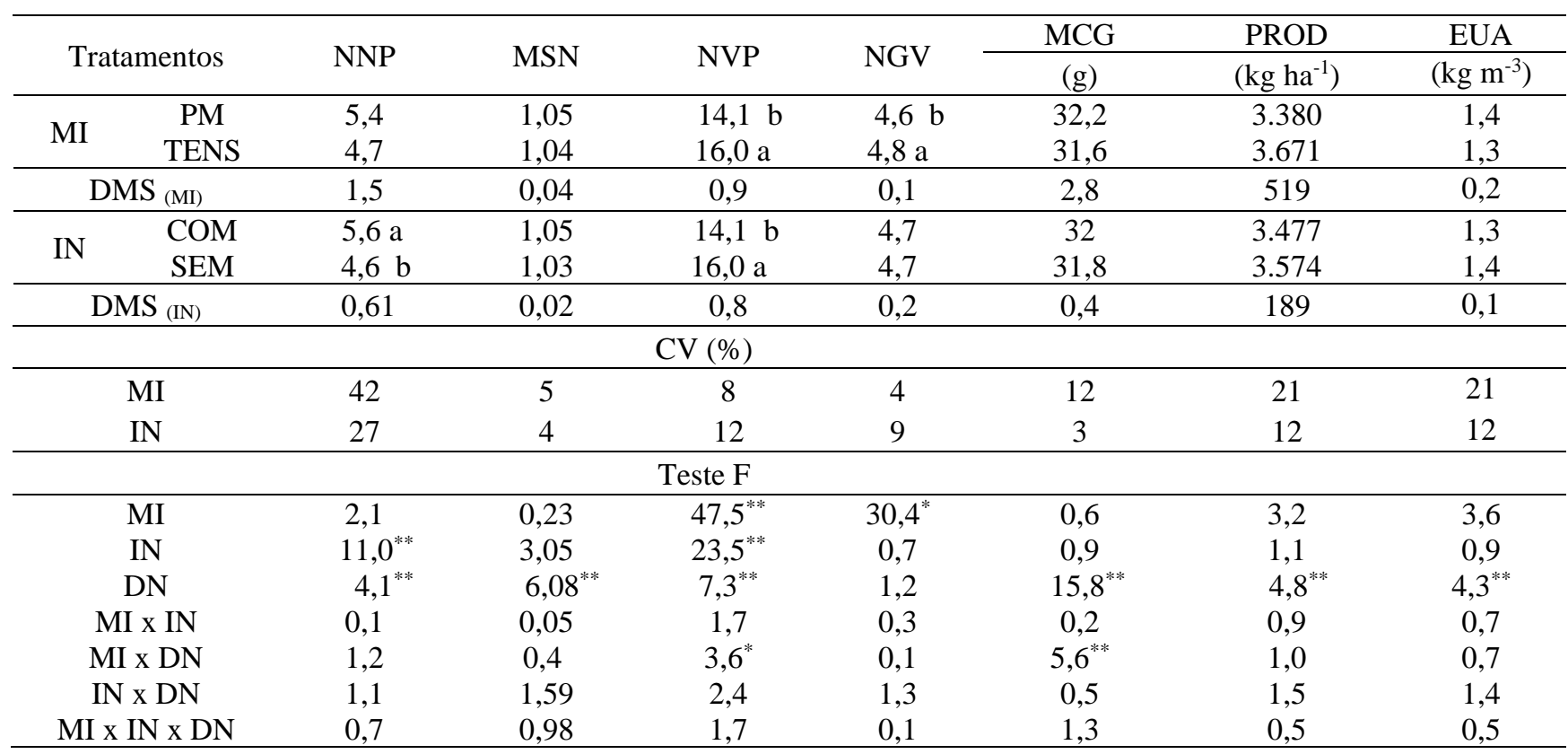

Médias seguidas de mesma letra nas colunas não diferem estatisticamente entre si ao nível de 5\% de probabilidade, PM - manejo de irrigação pelo método de Penman-Monteith, TENS - manejo de irrigação pelo método dos tensiômetros, DMS - diferença mínima significativa, CV - coeficiente de variação, NNP e MSN transformados em $\sqrt{ }(\mathrm{x}+1),{ }^{*}$ Significativo a $5 \%$ de probabilidade de erro pelo teste $\mathrm{F},{ }^{* *}$ Significativo a $1 \%$ de probabilidade de erro pelo teste F. Manejo de irrigação (MI), Inoculação (IN), Doses de nitrogênio (DN). Fonte: Autores.

O maior NVP foi obtido sem o uso de inoculante, enquanto não houve diferença entre os tratamentos para o NGV. Bastos et al. (2016), destacam que estes parâmetros referentes a produção, são diretamente influenciados pela disponibilidade de água durante o ciclo da cultura.

Em relação ao NNP e MSN, os resultados foram semelhantes para ambos os manejos de irrigação, isso porque esses fatores são pouco influenciados pela disponibilidade de água e estão diretamente relacionados a presença de inóculos, sejam nativos ou da inoculação com estipes conhecidas. Para Brito et al. (2015) a presença de população nativa de nódulos é um dos principais fatores que limitam o sucesso da inoculação de feijão comum.

No que se refere ao uso de Rhizobium tropici, nota-se que o NNP no tratamento com o uso de inoculante foi superior, entretanto mesmo sem o uso deste, observa-se a presença de nódulos nas plantas, comprovando a existência de estirpes nativas, fato que também pode ser observado na MSN, evidenciando essa limitação no sucesso da inoculação de feijão comum, observada por Brito et al. (2015).

Os diferentes manejos de irrigação e inoculação de sementes não influenciaram os resultados dos parâmetros MCG e PROD, sendo que a produtividade média obtida, de $3.525 \mathrm{~kg} \mathrm{ha}^{-1}$, foi $186,1 \%$ superior, quando comparado com a produtividade nacional, do feijão de inverno, que é de $1.232 \mathrm{~kg} \mathrm{ha}^{-1}$, e ainda supera em 13,6\% a maior produtividade registrada que foi de $3.101 \mathrm{~kg} \mathrm{ha}^{-1}$, no distrito federal (CONAB, 2019), destacando o potencial produtivo da região, no período de inverno, em condições de irrigação.

Esses resultados corroboram com os observados na EUA, mostrando que independentemente do método de irrigação adotado, ambos são eficientes e disponibilizam a quantidade de água demandada pela cultura e uma boa distribuição desta ao longo do ciclo.

O NNP diminuiu em função das doses de N (Figura 5). Enquanto que os resultados da MSN mostraram tendência 
similar, diminuindo até a dose de $150 \mathrm{~kg} \mathrm{ha}^{-1}$. Isso pode ser explicado pela limitação da nodulação e do crescimento destes, em razão da planta estar absorvendo nitrogênio oriundo do $\mathrm{N}$ aplicado. Resultados similares foram obtidos em trabalho realizado por Kaneko et al. (2010).

Figura 5. Ajustes de regressão para o número de nódulos por planta (NNP) (A), da massa seca de nódulos (MSN) (B), número de vagens por planta (NVP) (C), da massa de cem grãos (MCG) (D), a produtividade de grãos (PROD) (E) e para a eficiência no uso da água (EUA) (F).

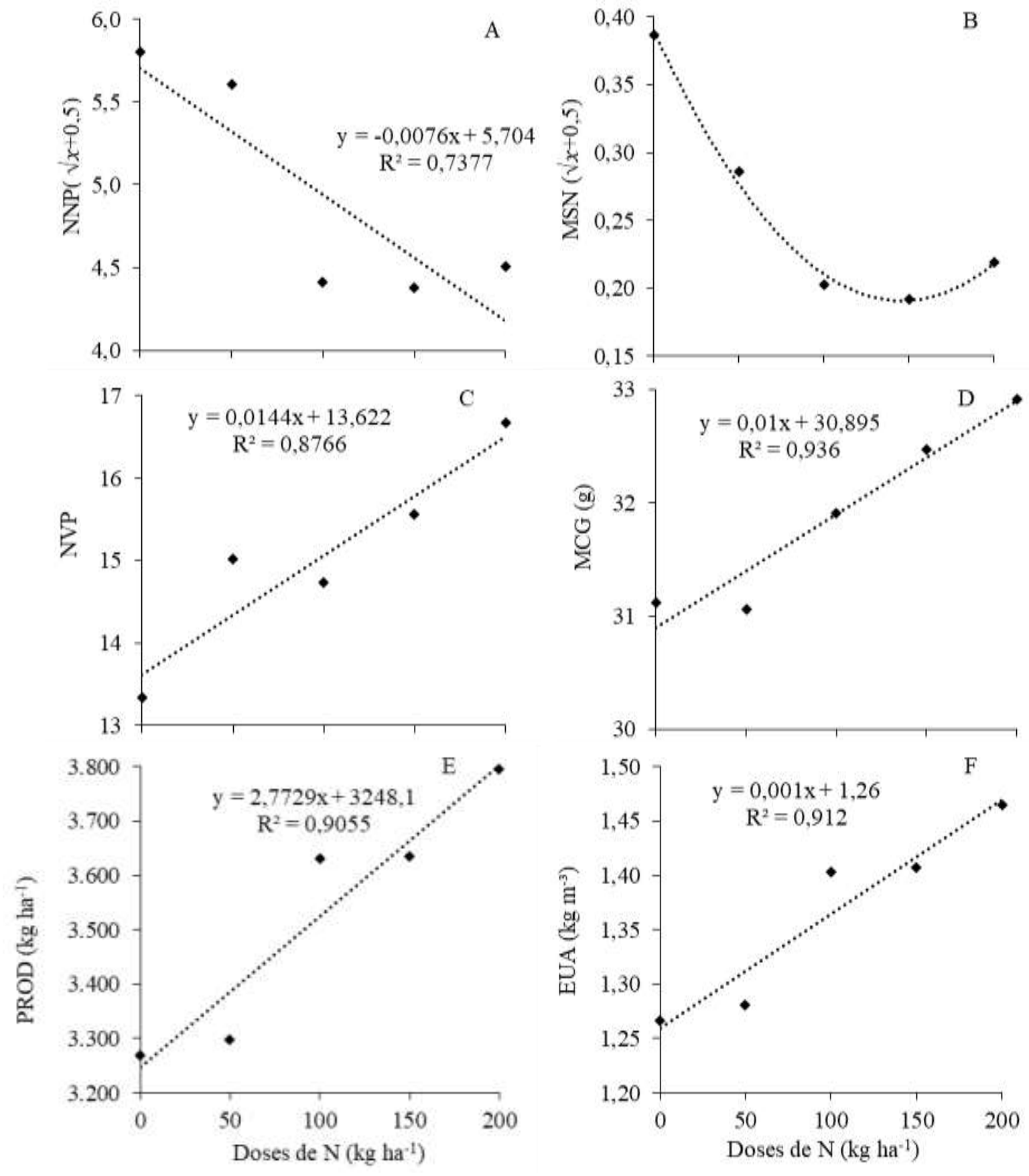

Fonte: Autores.

Em trabalho realizado por Santana et al. (2018), não houve influência das doses de N nas variáveis NVP e na MCG, já no presente trabalho ambas variáveis apresentaram incremento linear, conforme acréscimo de $\mathrm{N}$ em cobertura. Os resultados de PROD e de EUA apresentaram crescimento linear, para as doses de $\mathrm{N}$ em cobertura, não sendo possível definir a dose mais 
responsiva. Já Amaral et al. (2016), encontraram regressão quadrática para PROD e EUA, sendo os melhores resultados obtidos na dose de 136 e $101 \mathrm{~kg} \mathrm{ha}^{-1}$ respectivamente.

A EUA em função do aumento das doses de $\mathrm{N}$ variou de 1,26 a $1,46 \mathrm{~kg} \mathrm{~m}^{-3}$, semelhante ao trabalho de Pacheco et al. (2016). Esse aumento da EUA, em razão das maiores doses de N podem estar associados a influência deste nutriente no desenvolvimento das plantas, tanto na parte aérea quanto no maior aporte de raízes proporcionando desta forma, plantas com maior capacidade de absorção de água.

\section{Conclusão}

Ambos os manejos de irrigação proporcionam umidade do solo dentro dos limites da água facilmente disponível, sendo que, o manejo por Penman-Monteith estima menor lâmina de irrigação.

A inoculação de sementes com Rhizobium tropici não influencia na produtividade do feijoeiro.

A dose de $100 \mathrm{~kg}$ de $\mathrm{N} \mathrm{ha}^{-1} \mathrm{em}$ cobertura proporciona maiores produtividades de grãos e eficiência no uso da água pelo feijoeiro.

Devem ser desenvolvidos mais trabalhos com esta e outras variedades de feijão carioca na região, para obter informações sobre as doses de $\mathrm{N}$ e manejos de irrigação que proporcionam as maiores produtividades.

\section{Referências}

Allen, R. G., Pereira, L. S., Raes, D. \& Smith, M. (1998). Crop evapotranspiration: guidelines for computing crop requirements. FAO, 301p.

Alvares, C. A., Stape, J. L., Sentelhas, P. S., Gonçalves, J. L. M. \& Sparovek, G. (2014). Köppen's climate classification map for Brazil. Meteorologische Zeitschrift. 22(6), 711-728.

Amaral, C. B., Pinto, C. C., Flôres, J. A., Mingotte, F. L. C., Lemos, L. B. \& Fornasieri Filho, D. (2016). Produtividade e qualidade do feijoeiro cultivado sobre palhadas de gramíneas e adubado com nitrogênio em plantio direto. Pesquisa Agropecuaria Brasileira, 51(9), 1602-1609.

Barbosa, C. A. C., Leite, C. A. M., Santos, E. D., Soriani, R. \& Chagas, T. L. K. (2018). Envelhecimento acelerado e teste de germinação em sementes de feijão carioca (Phaseolus vulgaris). Revista Terra e Cultura: Cadernos de Ensino e Pesquisa, 34(1), $223-230$.

Bastos, F. J. D. C., Soares, F. A. L., Sousa, C. V., Tavares, C. J., Teixeira, M. B. \& Sousa, A. E. C. (2016). Common bean yield under water suppression and application of osmoprotectants. Revista Brasileira de Engenharia Agricola e Ambiental, 20(8), p. 697-701.

Bernardo, S., Soares, A. A. \& Mantovani, E. C. (2006). Manual de irrigação. (8a ed.), UFV, 625.

Brasil. (2009). Ministério da Agricultura e Reforma Agrária. Secretaria Nacional de Defesa Agropecuária. Departamento Nacional de Produção Vegetal. Coordenação de Laboratório Vegetal. Regras para análise de sementes. 365.

Brito, L. F., Pacheco, R. S., Souza Filho, B. F., Ferreira, E. P. B., Straliotto, R. \& Araújo, A. P. (2015). Resposta do feijoeiro comum à inoculação com rizóbio e suplementação com nitrogênio mineral em dois biomas brasileiros. Revista Brasileira de Ciencia do Solo, 39(4), 981-992.

Conab, Companhia Nacional de Abastecimento. (2019). Décimo segundo levantamento. Acompanhamento da safra brasileira de grãos 2012/2013, 6(1), 1-29.

Conceição, C. G., Robaina, A. D., Peiter, M. X., Parizi, A. R. C., Conceição, J. A. \& Bruning, J. (2018). Economically optimal water depth and grain yield of common bean subjected to different irrigation depths. Revista Brasileira de Engenharia Agrícola e Ambiental, $22(1), 482-487$.

Drugowich, M. I. \& D’auria, M. G. D. P. M. (2017). Uso racional da água na agricultura. CATI. 1(1), 44.

Fernandes, L. M. (2012). Retorno financeiro e risco de preço da cultura do feijão irrigado via pivô central na região noroeste de minas gerais. Informações Economicas, 42(1), 13 .

Fietz, C. R., Comunello E., Garcia, R. A, Flumignan, D. L., Silva, S. C. \& Melo, L. C. (2018). Demanda hídrica e risco climático de feijão superprecoce na região sul de Mato Grosso do Sul. Embrapa, 1(45), 13.

Kaneko, F. H., Arf, O., Gitti, D. C., Arf, M. V., Ferreira, J. P. \& Buzetti, S. (2010). Mecanismos de abertura de sulcos, inoculação e adubação nitrogenada em feijoeiro em sistema plantio direto. Bragantia, 69(1), 125-133.

Mapa, Ministério da Agricultura, Pecuária e Abastecimento. (2013). Instrução Normativa n 45, de 17 de setembro de $2013.13(45)$, 1-38.

Miranda, M. N. \& Campelo Junior, J. H. (2010). Soma térmica para o subperíodo semeadura-maturação de feijão cv. Carioca em colorado do oeste. Rondônia. Pesquisa Agropecuária Tropical. 40(2), 180-185. 
Research, Society and Development, v. 10, n. 8, e56910817437, 2021

(CC BY 4.0) | ISSN 2525-3409 | DOI: http://dx.doi.org/10.33448/rsd-v10i8.17437

Oliveira, L. F. C., Oliveira, M. G. C., Wendland, A., Heinemann, A. B., Guimarães, C. M., Enderson, P. B. F., Quintela, E. D., Barbosa, F. R., Carvalho, M. C. S., Lobo Junior, M., Silveira, P. M. \& Silva, S. C. (2018). Conhecendo a fenologia do feijoeiro e seus aspectos fitotécnicos. (2a ed.), Embrapa.

Pacheco, A., Lopes, A. S., Oliveira, G. Q., França, A. \& Silva, L. E. (2016). Diferentes métodos de manejo de irrigação e doses de adubação nitrogenada nos componentes de produtividade e eficiência no uso da água. Revista Agrarian, 9(33), 263-273.

Pereira, V. G. C., Gris, D. J., Marangoni, T., Frigo, J. P., Azevedo, K. D. \& Grzesiuck, A. E. (2014). Exigências agroclimáticas para a cultura do feijão (Phaseolus vulgaris L.). Revista Brasileira de Energias Renováveis, 3(1), 32-42.

Ramires, R., Lima, S., Simon, C.A., Contardi, L. M., Alvarez, R. C. F. \& Brasil, M. (2018). Inoculação com rizóbio associado ao manejo da adubação nitrogenada em feijão comum. Colloquium Agrariae, 14(1), 49-57.

Rebeschini, A. C., Mazzuchelli, R. C. L., Araujo, A. S. F. \& Araujo, F. F. (2014). Nitrogen application and inoculation with Rhizobium tropici on common bean in the fall/winter. African Journal of Agricultural Research, 9(42), 3156-3163.

Sabundjian, M. T., Arf, O., Kaneko, F. H. \& Ferreira, J. P. (2013). Nitrogen fertilization on common bean in succession to maize and Urochloa ruziziensis single cropping and intercropping. Pesquisa Agropecuária Tropical, 43(3), 292-299.

Santana, M. V., Ferreira, B. S., Santos, T. T. M., Silva, J. O., Tavares, C. J. \& Dornelles, M. S. (2018). Redução da adubação nitrogenada em cobertura de feijoeiro com Rhizobium tropici. Global Science and Technology, 1(1), 88-100.

Schiavo, J. A., Pereira M. G., Miranda, L. P. M., Dias Neto, A. H. \& Fontana, A. (2010). Caracterização e classificação de solos desenvolvidos de arenitos da formação Aquidauana-MS. Revista Brasileira de Ciência do Solo. 34(1), 881-889.

Silva, E. F., Marchetti, M. E., Souza, L. C. F., Mercante, F. M., 19 Rodrigues, E. T. \& Vitorino, A. C. T. (2009). Inoculação do feijoeiro com Rhizobium tropici associada à exsudato de Mimosa flocculosa com diferentes doses de nitrogênio. Bragantia. 68(2).

Silveira, P. M., Stone, L. F. \& Moreira, J. A. A. (2001). Manejo da água de irrigação. In: Silveira, P. M. \& Stone, L. F. Irrigação do feijoeiro. Santo Antônio de Goiás-GO: EMBRAPA, 1(1), 139-170.

Sousa, D. M. G. \& Lobato, E. (2004). Cerrado correção do solo em adubação. (2a ed.), Embrapa Informação Tecnológica, 416.

Teixeira, G. C. Da S., Stone, L. F. \& Heinemann, A. B. (2015). Eficiência do uso da radiação solar e índices morfofisiológicos em cultivares de feijoeiro. Pesquisa Agropecuaria Tropical, 45(1), 9-17.

Testezlaf, R. (2017). Irrigação: métodos, sistemas e aplicações. Unicamp/FEAGRI, 215.

Viçosi, K. A., Ferreira, A. A. S., Oliveira, L. A. B. \& Rodrigues, F. (2017). Estresse hídrico simulado em genótipos de feijão, milho e soja. Journal of Neotropical Agriculture, 4(5), 36-42. 